Volume 7, No.5, September - October 2018

International Journal of Information Systems and Computer Sciences

Available Online at http://warse.org/IJISCS/static/pdf/file/ijiscs02752018.pdf

https://doi.org/10.30534/ijiscs/2018/02752018

\title{
Development of Fuzzy-Based Expert System for Stroke Prevention
}

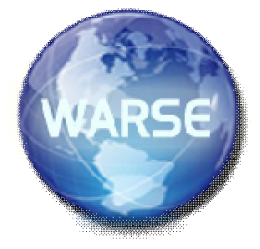

\author{
Olatunji K. A. ${ }^{1}$, Oguntimilehin A. ${ }^{2}$ ， Babalola G. O. ${ }^{3}$ \\ ${ }^{1}$ Afe Babalola University Ado Ekiti, Ekiti State, Nigeria \\ olatunjika@abuad.edu.ng \\ ${ }^{2}$ Afe Babalola University Ado Ekiti, Ekiti State, Nigeria \\ ebenabiodun2@yahoo.com \\ ${ }^{3}$ Afe Babalola University Ado Ekiti, Ekiti State, Nigeria \\ gbemibabz@abuad.edu.ng
}

\begin{abstract}
A time has come whereby the rural dwellers need to be aware of the various health challenges that are facing them, and one of the best ways to reach this group of people is through a Mobile System because the majority of rural dwellers have died or become a burden to their families and society at large due to lack of beneficial information and standard health care facilities. Government of the day concentrates more in developing the urban areas rather than the rural areas. Most prominent disease that comes unexpectedly and the general public (especially the rural dwellers) is not usually aware of its symptoms is Stroke. Stroke could be prevented and its risk factors have been identified. Through a stroke prevention expert system, the user could be made more aware of stroke risks and symptoms. An expert system would be able to direct and motivate users to keep themselves healthy therefore preventing occurrence of stroke.

In this research work, Fuzzy-Based Expert System for stroke prevention was designed. The model will be implemented using JAVA along with Extended Markup Language as the front-end. Android Studio Integrated Development Environment will be used for interface design of mobile forms and code editing. Embedded SQLite will serve as a backend.
\end{abstract} Key Words: Diagnosis, Expert Systems, Fuzzy Logic, Mobilecertain situations, whether either the case is quite complex Application, Stroke.

\section{INTRODUCTION}

Expert system is a knowledge intensive computer program that captures the expertise of a human in domains of knowledge. Medical Expert systems are the common type of Artificial Intelligence in medical in routine clinical use. They contain medical knowledge, usually about a very specifically defined task, and are able to reason with data from individual patients to come up with reasoned conclusions [1].

The rapid integration of mobile devices into clinical practice has, in part, been driven by the rising availability and quality of medical software applications, or "apps". Apps are software programs that have been developed to run on a computer or mobile device to accomplish a specific purpose. Faster processors, improved memory, smaller batteries, and highly efficient open-source operating systems that perform complex functions have paved the way for the development of a flood of medical mobile device apps for both professional and personal use [10]. The new trend gaining momentum in recent years is use of mobile devices as an integral part of their IT infrastructure; mobile based applications allow location independence in contrast to the static nature of desktop applications. Mobile phones combine the ability of portability and networking which makes them a reasonable option for graphical user interface (GUI) of expert systems.

Today, there is need of developing the expert system for stroke prevention (also known as Cerebrovascular accident) because it is one of the leading of death and adult disability worldwide and it amounted to $15.4 \%$ of total deaths which roughly calculated to one person among seven. It is noted that by the knowledge of personal stroke risk is a beginning step in preventing stroke. [2] and [8] calculated that costs to prevent stroke are significantly less than to treat one. In and there is no medical experts readily available for patients, expert systems are useful. Health provision is challenging due to costs required as well as well as various social, cultural, political and economic conditions.

\section{REVIEWED OF THE RELATED WORK}

The following related researches were reviewed as follows: 
Olatunji K. A et al., International Journal of Information Systems and Computer Sciences, 7(5), September - October 2018, 25-33

[7] developed a Computerized Algorithm for Etiologic Classification of Ischemic Stroke (SSS-TOAST)-The Causative Classification of Stroke System. In their research they realized that reliable classification of stroke, however, is a complex task because stroke is a heterogeneous disorder with multiple potential mechanisms. Inter-rater agreement decreases when attempts are made to classify strokes with multiple mechanisms into specific etiologic classes in the absence of evidence-based strategies.

The CCS incorporates clinical, epidemiological (quantitative primary stroke risk estimates), and diagnostic data to determine stroke subtype in 5 major categories: large artery atherosclerosis, cardio-aortic embolism, small artery occlusion, other causes, and undetermined causes. The undetermined group is further divided into cryptogenic embolism, other cryptogenic, incomplete evaluation, and unclassified categories. In the CCS, each etiologic category is subdivided based on the weight of evidence as "evident," "probable," or "possible". A mechanism is deemed "evident" only if the available data indicate that it is the sole potential mechanism conforming to 1 of the etiologic categories. When there are $>1$ "evident" stroke mechanisms, the system assigns a "probable" stroke mechanism based on specific characteristics that make one mechanism more probable than the others. In the absence of any "evident" cause, a search is made for "possible" mechanisms that carry a lower or less-well defined risk for stroke. Their current version of the CCS software offers a 5-patient training module based on abstracted information on clinical and diagnostic findings. Differences in interpretation of test results were a source of disagreement among examiners. This is a distinction that is difficult to make from abstracted test reports unless the reporting physician's diagnosis is explicitly stated. The diagnosis requires individual physician's primary assessment based on location, shape, and composition of stenosis, as well as coexisting changes in other vascular sites.

Web-based Tool for Dynamic Functional Outcome after Acute Ischemic Stroke and Comparison with Existing Models was designed and implemented by [13]. They found out that Stroke is one of the leading causes of death and adult disability worldwide and acute ischemic stroke (AIS) accounts for about $85 \%$ of all stroke cases. Although few recently proposed risk models could provide dynamic prognostic information at multiple time points after AIS, they mainly focused on mortality instead of functional status. In real-world practice, patients, family members, clinicians and researchers usually concern about not only the likelihood of survival, but also the likelihood of survival with function recovery after AIS.

The DFS-AIS was developed based on the China National Stroke Registry (CNSR), in which eligible patients were randomly divided into derivation (60\%) and validation (40\%) cohorts. Standardized case report form (CRF) was used for data collection in the CNSR network. The relevant data was extracted from the medical records by trained research coordinators. Data from each CRF were manually checked for completeness, correct coding, and proper application of diagnostic algorithm by a research specialist from an independent contract research organization. In the study, they analyzed the following candidate variables: (1) demographics (age and gender); (2) stroke risk factors: hypertension (history of hypertension or anti-hypertensive medication use), diabetes mellitus (history of diabetes mellitus or anti-diabetic medication use), dyslipidemia (history of dyslipidemia or lipid-lowering medication use), atrial fibrillation (history of atrial fibrillation or documentation of atrial fibrillation on admission), coronary heart disease, history of stroke/TIA, current smoking, and excess alcohol consumption ( $\geq 2$ standard alcohol beverages per day); (3) pre-existing comorbidities: congestive heart failure, valvular heart disease, peripheral artery disease, chronic obstructive pulmonary disease (COPD), hepatic cirrhosis, peptic ulcer or previous gastrointestinal bleeding (GIB), renal failure, Alzheimer's disease/dementia, and cancer; (4) pre-stroke dependence (modified Rankin Scale score $\geq 3$ ); (5) pre-admission antithrombotic medications: anticoagulant with warfarin (for atrial fibrillation) or antiplatelet medication (aspirin, clopidogrel, or extended release dipyridamole combined with aspirin); (6) pre-admission statins using; (7) transportation mode to hospital (dichotomized as by emergency medical system [EMS] or private transportation [such as by taxi or private car]); (8) Time from onset to hospital arrival (hours); (9) admission systolic and diastolic blood pressure $(\mathrm{mmHg})$; (10) admission stroke severity based on National Institutes of Health Stroke Scale (NIHSS) score; (11) stroke subtypes according to the Oxfordshire Community Stroke Project (OCSP) criteria (Bamford et.al, 1993), where AIS was classified into partial anterior circulation infarct (PACI), total anterior circulation infarct (TACI), lacunar infarction (LACI), and posterior circulation infarct (POCI). We did not use TOAST subtype because it requires the results of investigations that usually are not available at the time of admission for many patients; (12) admission blood glucose $(\mathrm{mmol} / \mathrm{L})$.

The modified Rankin Scale (mRS) was used to assess functional outcome at different time points (discharge, 3month, 6-month and 1-year) after AIS. Treating physician evaluated patients' functional status at discharge. A central follow-up for functional status at 3-month, 6-month and 1year after onset was made by telephone interview by trained interviewers based on a standardized interview protocol. Good functional outcome was defined as $m R S \leq 2$ at discharge, 3-month, 6-month, and 1-year after AIS, respectively. Independent predictors of each outcome measure were obtained using multivariable logistic regression.

The major limitation of the research is that there might be complex genetic, social, culture, economic factors as well as regional management philosophies and preferences that are difficult to account for when prognostic models are 
Olatunji K. A et al., International Journal of Information Systems and Computer Sciences, 7(5), September - October 2018, 25-33

developed or applied to a distinct population and it is not mobile based.

Diagnosis and Treatment of Patients with Stroke in a Mobile Stroke Unit versus in Hospital: a Randomized Controlled Trial was presented by [15]. It was discovered that only 2$5 \%$ of patients who have a stroke receive thrombolytic treatment, mainly because of delay in reaching the hospital and about $90 \%$ of all strokes are due to cerebral ischaemia, with the remainder due to cerebral haemorrhage. The only approved treatment for ischaemic stroke is recanalisation of occluded arteries by thrombolysis with alteplase within the very first hours of symptom onset. However, implementation of recanalising therapy within this narrow therapeutic window is difficult to achieve in clinical practice because neurological examination, imaging, and laboratory analyses are needed so that haemorrhagic stroke and other contraindications to thrombolysis can be excluded.

They carried out a randomized single-centre controlled trial to compare the time from alarm (emergency call) to therapy decision between mobile stroke unit (MSU) and hospital intervention. Patients between the age of 18-80 years were involved that have one or more stroke symptoms that started within the previous $2.5 \mathrm{~h}$. In accordance with their weekwise randomization plan, patients received either prehospital stroke treatment in a specialized ambulance (equipped with a CT scanner, point-of-care laboratory, and telemedicine connection) or optimized conventional hospital-based stroke treatment (control group) with a 7 day follow-up. Allocation was not masked from patients and investigators. Since their primary endpoint was time from alarm to therapy decision, it was analyzed with the MannWhitney $U$ test. And the secondary endpoints included times from alarm to end of CT and to end of laboratory analysis, number of patients receiving intravenous thrombolysis, time from alarm to intravenous thrombolysis, and neurological outcome. They also assessed safety endpoints. The major set back for this research are lack of power for the process, other secondary endpoints in the subpopulation of stroke patients, the relatively short follow-up time of outcomerelated secondary endpoints, the potential effects of previous disability and the absence of masking in the assessment.

[9] developed a MICROSTROKE Expert System for Stroke Type Diagnosis to categorize and diagnose stroke types based on clinical information and it is intended as a practical aid for physicians not fully familiar with the diagnosis of stroke types. They realized that for the bedside assessment of stroke type, knowledge of the frequency distributions of signs, symptoms, and ecological data associated with the different stroke types can be of prime importance. And also much research effort has been devoted to the development of expert systems to cope with complex medical decisionmaking during the last decade.

MICROSTROKE includes three knowledge data bases. The first contains frequency distributions of clinical and ecological parameters for the stroke types thrombosis, embolus, lacune, intracerebral hemorrhage (ICH), and subarachnoid hemorrhage (SAH). Data are derived from the Stroke Data Bank [6], the Michael Reese Stroke Registry [4], and the Harvard Cooperative Stroke Registry [11]. The frequency distribution tables contain relative frequencies of single items (symptoms or historical data) or of a set of alternative symptoms for all stroke patients and for each stroke type, respectively. A second knowledge data base is implemented using rule-based information coding. Rulebased systems depend on the hypotheses that expert knowledge consists of many independent, situation specific rules and that computers can simulate expert reasoning by stringing these rules together in chains of deduction. The "if" part of a rule (the premise) contains the pattern or attributes that must be matched for the rule to be used. The "then" part (the conclusion) contains an assertion to be made when the premise is satisfied. A typical rule is "If there is hypertension at onset and early course of deficit is gradual smooth progression of symptoms, then display warning for ICH." Certain rules include combinations of symptoms that are associated with a high probability of intracranial hemorrhage. At present, MICROSTROKE matches the premises of rules with data from a current patient to prompt a warning for ICH, SAH, or both if they apply.

The third knowledge data base consists of exclusively text information, used by the tutorial module of MICROSTROKE and stored as an American Standard Code for Information Interchange (ASCII) file. This knowledge data base serves only educational purposes and has no influence on calculations of stroke type diagnostic probabilities. MICROSTROKE acquires knowledge by interactively asking the physician, user for details of the patient's history, information about the onset of stroke, and accompanying symptoms in a questionnaire comprising items for which frequency distribution tables in different types of stroke are available. The answers accepted are yes, no, unknown, or an option if a multiple-choice question is presented.

The inference engine of any expert system is the computer program that provides its general problem solving capabilities. The inference engine is separated from the collection of domain knowledge, the knowledge database; MICROSTROKE'S inference engine calculates probabilities of different stroke types using modified Bayesian inference techniques. Each stroke type is attributed an account. Starting from initial accounts representing the a priori odds for the five stroke types, accounts are recalculated after each question depending on the physician-user's answer. The order of the questions presented depends on the MICROSTROKE mode selected. If there is no laboratory data available, intracranial hemorrhage cannot be excluded, and MICROSTROKE'S first goal is to detect signs of ICH or $\mathrm{SAH}$. If intracranial hemorrhage has already been excluded, for example, by CT scan and lumbar puncture, data are acquired to differentiate ischemicstroke types. The accounts are displayed as probabilities of stroke types by multiplying each with a constant, yielding an account sum of 100 . In 
Olatunji K. A et al., International Journal of Information Systems and Computer Sciences, 7(5), September - October 2018, 25-33

order for the system to be efficient and function well, there is need to expand the knowledge base, incorporate its own experience in its stroke registry, modify underlying inference techniques, made it a mobile based system and implements the system.

Expert-System Based Medical Stroke Prevention was designed and implemented by [2]. It was revealed through past research that stroke always comes unexpected and the general public is not usually aware of its symptoms. Individuals who have had their first stroke with permanent damage could become an economic burden to their family and a social burden to the society due to their unproductive nature. Stroke could be prevented and its risk factors have been identified and medical stroke is the most common cause of death amounted to $15.4 \%$ of total deaths which roughly calculated to one person among seven. The stroke prevalence in 2007 is 800 occurrences every hundred thousand citizens.

The major components of expert system are knowledge base, inference engine and the conclusion which is the output from the expert system containing prevention information and other suggestions to decrease stroke risk factors. The knowledge base design includes blood pressure, body mass index, cigarette consumption and physical activity. Knowledge base for blood pressure is based on systolic blood pressure range (pressure when heart contracts) and diastolic blood pressure range (pressure when heart is filled with blood). Stroke risk increases twice every $20 / 10 \mathrm{mmHg}$ [5]. Body Mass Index (BMI) is a simple index of weightforheight that is commonly used to classify underweight, overweight and obesity in adults [12]. It is defined as the weight in kilograms divided by the square of the height in metres ( $\mathrm{kg} \mathrm{m}-2)$. On consumption of cigarettes, the pattern is divided into several categories. While there have been no references on correlation of the number of cigarettes and stroke risk, it is assumed that the target is no consumption. Advice is given based on the current level of consumption. The next target would be a lower level consumption than before and advice is subsequently given until no consumption is achieved. [14] recommends $3.5 \mathrm{~h}$ per week of moderate activity to reduce stroke risks. This is equivalent of 0 .

An inference engine that provides stroke risk level based on information provided by the user is built. A score of +1 is given to a stage increase compared to recommended baseline levels for reducing stroke risks and a score of- 1 is given to a lower stage below baseline levels. Prevention advice given to high blood pressure is to reduce blood pressure levels by using medication or other recommended treatment from their physicians. Prevention advice for body mass index is to lower their body mass index to a stage below current level. Prevention advice for cigarette consumption is based on lowering consumption to a stage lower than current level. Physical activity advice is given to increase physical activity amount in the next stage before current level.

This research should have been mobile-based because of the difficulties of necessary equipment and facilities involves in the treatment of stoke available in rural communities health centre. Also the research should never be recommended as a replacement for proper medical treatment.

\section{DESIGN OF A MOBILE BASED EXPERT SYSTEM FOR STROKE PREVENTION}

The design of this research work involved data collection, review of existing work on the subject matter, development of prevention system for stroke. The architecture of the proposed prevention system is presented in Figure 1. The system comprises of mobile forms and embedded database.

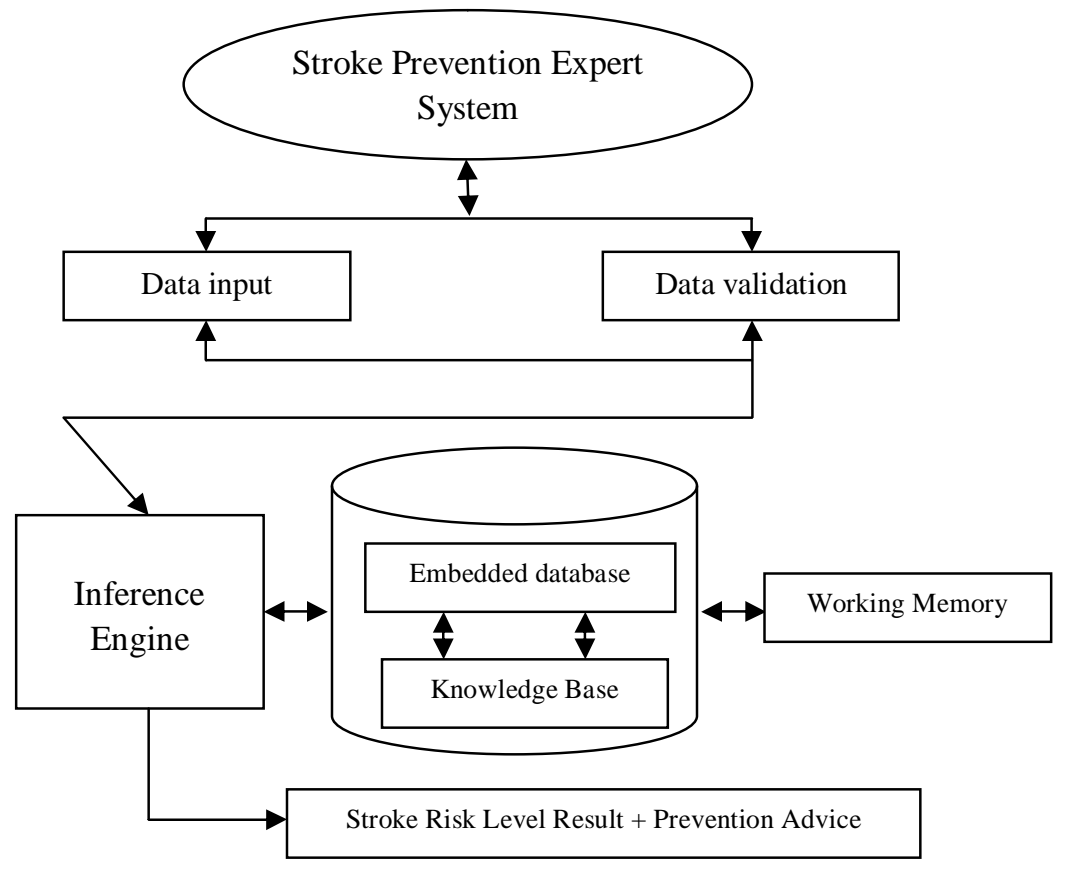

Figure 1: Architecture of the proposed System 
Olatunji K. A et al., International Journal of Information Systems and Computer Sciences, 7(5), September - October 2018, 25-33

\subsection{Data Collection}

Online materials were consulted and data was collected from Ekiti State Teaching Hospital, Ado Ekiti (EKSUTH). They are categorized into four as follows:

a. Blood Pressure

i. Systolic : Pressure when heart contracts

ii. Diastolic : Pressure when heart is filled with blood

b. Body mass index: Body Mass Index (BMI) is a simple index of weight for-height that is commonly used to classify underweight, overweight and obesity in adults. It is defined as the weight in kilograms divided by the square of the height in metres $(\mathrm{kg} \mathrm{m}-2)$.

i. Height

ii. Weight

c. Cigarettes Smoked

d. Physical Activity: $3.5 \mathrm{~h}$ per week of moderate activity to reduce stroke risks is recommended.
i. Hours
ii. Mins

\subsection{Expert System}

From the records of the patients checked, a total of thirteen (13) risk factors or causes of stroke were observed. The interaction with the medical experts lead to the four major risk factors that is common to all patient's records. The expert system for prevention of Stroke was designed using the fuzzy logic approach. In this system, the four major risk factors which are Smoking (SM), Body Mass Index (BMI),
Blood Pressure (BP), and Exercise (EX) were taken as input parameters to the expert system and the "Stroke Risk (SK)" was the output parameter. The resultant stroke risk was based on fuzzy rules that were developed for the expert system. These rules served as the knowledge base of the mobile expert system.

\subsubsection{Fuzzy Logic}

Fuzzy logic in this system is made up of 3 main modules. These are: Fuzzification module, Fuzzy Inference module and the De-Fuzzification module. The fuzzy logic Model is presented in figure 2.

\section{A) Fuzzification}

Fuzzification is a process that determines the degree of membership to the fuzzy set based on fuzzy membership function. Smoking (SM), Body Mass Index (BMI), Blood Pressure (BP), and Exercise (EX) parameters constitute the fuzzy logic input variables used to generate the fuzzy logic model, and the output parameter is Stroke Risk (SK). These input parameters are used to map the output value specified in the individual rules to an intermediate output measuring fuzzy sets (Blood Pressure: Low BP, Normal BP, High BP, VeryHigh BP and VeryVeryHigh BP, Body Mass Index: Low BMI, Normal BMI, High BMI, VeryHigh BMI, and VeryVeryHigh BMI, Smoking: No SM, Low SM, Moderate SM, High SM, and VeryHigh SM, and Exercise: No EX, Low EX, Medium EX, Normal EX and High EX). The classification into classes for each attribute is as shown in the tables 1-4.

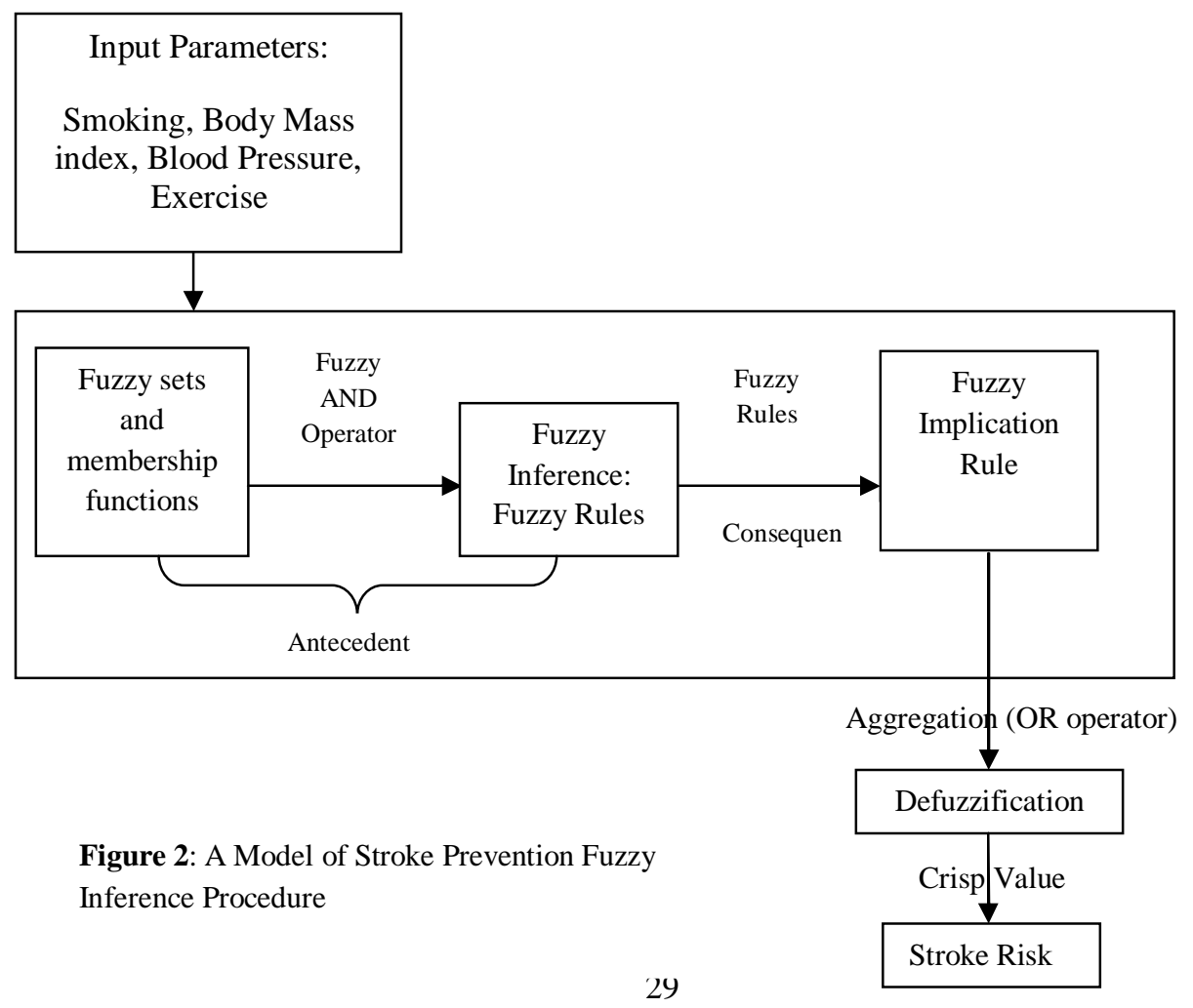


Olatunji K. A et al., International Journal of Information Systems and Computer Sciences, 7(5), September - October 2018, 25-33

Table 1: Classification for Body Mass Index

\begin{tabular}{|l|l|l|}
\hline BMI Value Range & Classification & Comment \\
\hline$<18.50$ & Low & Underweight \\
\hline $18.50-24.99$ & Normal & Normal Weight \\
\hline $25.00-29.99$ & High & Overweight \\
\hline $30.00-34.99$ & VeryHigh & Obese I \\
\hline$>=35.00$ & VeryVeryHigh & Obese II \\
\hline
\end{tabular}

Table 2: Classification for Blood Pressure

\begin{tabular}{|l|l|l|}
\hline Systolic Range & Diastolic Range & Classification \\
\hline$<90.0$ & $<59.0$ & Low \\
\hline $90.0-120.0$ & $60.0-80.0$ & Normal \\
\hline $121.0-139.0$ & $80.0-89.0$ & High \\
\hline $140.0-159.0$ & $90.0-99.0$ & VeryHigh \\
\hline$>=160.0$ & $>=100.0$ & VeryVeryHigh \\
\hline
\end{tabular}

Table 3: Classification for Smoking

\begin{tabular}{|l|l|l|}
\hline $\begin{array}{l}\text { Cigarette per } \\
\text { day }\end{array}$ & Classification & Comment \\
\hline 0 & No & None \\
\hline $1-6$ & Low & Low Consumption \\
\hline $7-12$ & Moderate & $\begin{array}{l}\text { Moderate } \\
\text { Consumption }\end{array}$ \\
\hline $13-24$ & High & High Consumption \\
\hline$>=24$ & VeryHigh & $\begin{array}{l}\text { Heavy } \\
\text { Consumption }\end{array}$ \\
\hline
\end{tabular}

Table 4: Classification for Exercise

\begin{tabular}{|l|l|l|}
\hline Hours per week & Classification & Comment \\
\hline 0 & No & No Exercise \\
\hline$>0-1.4$ & Low & Low \\
\hline$>1.4-2.4$ & Medium & Low-Medium \\
\hline$>2.4-3.4$ & Normal & Moderate \\
\hline$>=3.5$ & High & Recommended \\
\hline
\end{tabular}

\section{B) Membership Functions}

Membership function in fuzzy logic represents the degree of truth as an extension of valuation. For any set $\mathrm{X}$, a membership function on $\mathrm{X}$ is any function from $\mathrm{X}$ to the real unit interval $[0,1]$. Membership functions on $X$ represent fuzzy subsets of $X$. The membership function which represents a fuzzy set $\mathrm{A}$ is usually denoted by $\mu A$. For an element $x$ of $\mathrm{X}$, the value $\mu A(x)$ is called the membership degree of $x$ in the fuzzy set $\mathrm{A}$. The membership degree $\mu A(x)$ quantifies the grade of membership of the element $x$ to the fuzzy set $A$. The value 0 means that $x$ is not a member of the fuzzy set; the value 1 means that $x$ is fully a member of the fuzzy set. The values between 0 and 1 characterize fuzzy members, which belong to the fuzzy set only partially. The Triangular membership function was used to show the membership functions for the input parameters. Using the triangular membership, Equations $1-6$ show the membership functions used for the fuzzification of attribute BMI.

$$
\begin{aligned}
& B M I(x)=\left\{\begin{array}{cc}
0 & \text { if } x<1 \\
\frac{x-1}{18.5-1} \text { if } 1 \leq x<18.5 \\
\frac{24.99-x}{24.99-18.5} \text { if } 18.5 \leq x \leq 24.99 \\
\frac{29.99-x}{29.99-24.99} \text { if } 25 \leq x \leq 29.99 \\
\frac{34.99-x}{34.99-29.99} \text { if } 30 \leq x \leq 34.99 \\
1 \quad \text { if } x \geq 35
\end{array}\right. \\
& \mu_{\text {Low }}=\left\{\begin{array}{lr}
0 & \text { if } x<1 \\
\frac{x-1}{8.25} \text { if } & 1 \leq x<9.25 \\
\frac{18.5-x}{9.25} \text { if } & 9.25 \leq x \leq 18.5 \\
0.40 & \text { if } x>18.5
\end{array}\right. \\
& \mu_{\text {Normal }}=\left\{\begin{array}{lr}
\frac{x-18.5}{3.25} \text { if } & 18.5 \leq x<21.75 \\
\frac{18.5-x}{3.25} \text { if } & \text { if } x<1.75 \leq x \leq 24.99 \\
0.60 & \text { if } x>24.99
\end{array}\right. \\
& \mu_{\text {High }}=\left\{\begin{array}{lr}
0 & \text { if } x<25 \\
\frac{x-25}{2.50} \text { if } & 25 \leq x \leq 27.50 \\
\frac{25-x}{2.50} \text { if } & 27.50 \leq x \leq 29.99 \\
0.75 & \text { if } x \geq 30.00
\end{array}\right. \\
& \mu_{\text {VeryHigh }}=\left\{\begin{array}{lr}
0 & \text { if } x<30.00 \\
\frac{x-30}{2.5} \text { if } & 30.00 \leq x<32.50 \\
\frac{34.99-x}{2.5} \text { if } 32.5 \leq x \leq 34.99 \\
0.85 & \text { if } x>35
\end{array}\right. \\
& \mu_{\text {VeryVeryHigh }}= \begin{cases}0 & \text { if } x<35 \\
1 & \text { if } x>35\end{cases}
\end{aligned}
$$

The fuzzy class is dependent on two attributes, which are systolic and diastolic values, however, both values are directly proportional hence, either of the two attributes can be used.Using the triangular membership, Equations 7-12 show the membership functions used for the fuzzification of attribute BP (Diastolic).

$$
B P(x)=\left\{\begin{array}{rr}
0 & \text { if } x<1 \\
\frac{x}{59} \text { if } 0<x<59 & \\
\frac{80-x}{20} \text { if } 60 \leq x \leq 80 \\
\frac{89-x}{9} \text { if } 80<x \leq 89 \\
\frac{99-x}{9} \text { if } 90 \leq x \leq 99 \\
1 \quad \text { if } x \geq 100
\end{array}\right.
$$


Olatunji K. A et al., International Journal of Information Systems and Computer Sciences, 7(5), September - October 2018, 25-33

$$
\begin{aligned}
& \mu_{\text {Low }}=\left\{\begin{array}{cr}
0 & \text { if } x<1 \\
\frac{x-1}{29} \text { if } & 0<x<29 \\
\frac{59-x}{30} \text { if } & 29 \leq x \leq 59 \\
0.45 & \text { if } x>59
\end{array}\right. \\
& \mu_{\text {High }}=\left\{\begin{array}{cr}
0 & \text { if } x<12 \\
\frac{x-12}{6} \text { if } & 12 \leq x \leq 18 \\
\frac{24-x}{6} \text { if } & 18<x \leq 24 \\
0.97 & \text { if } x>24
\end{array}\right. \\
& \mu_{\text {Normal }}=\left\{\begin{array}{rr}
0 & \text { if } x<60 \\
\frac{x-60}{10} \text { if } & 60 \leq x<70 \\
\frac{80-x}{10} \text { if } & 70 \leq x \leq 80 \\
0.65 & \text { if } x>80
\end{array}\right. \\
& \mu_{\text {VeryHigh }}= \begin{cases}0 & \text { if } x<24 \\
1 & \text { if } x>24\end{cases}
\end{aligned}
$$$$
\mu_{\text {High }}=\left\{\begin{array}{lr}
0 & \text { if } x<80 \\
\frac{x-280}{4} \text { if } & 80 \leq x \leq 84 \\
\frac{89-x}{4} \text { if } & 85 \leq x \leq 89 \\
0.78 & \text { if } x>89
\end{array}\right.
$$$$
\mu_{\text {VeryHigh }}=\left\{\begin{array}{cr}
0 & \text { if } x<89 \\
\frac{x-90}{4} \text { if } & 90 \leq x<94 \\
\frac{99-x}{4} \text { if } & 95 \leq x \leq 99 \\
0.83 & \text { if } x>99
\end{array}\right.
$$$$
\mu_{\text {VeryVeryHigh }}= \begin{cases}0 & \text { if } x<100 \\ 1 & \text { if } x \geq 100\end{cases}
$$

Using the triangular membership, Equations 19-24 show the membership functions used for the fuzzification of attribute EX.

$$
E X(x)=\left\{\begin{array}{cc}
0 & \text { if } x=0 \\
\frac{x-1}{18.4-1} \text { if } 0<x \leq 1.4 \\
\frac{24.99-x}{24.99-18.5} \text { if } 1.4<x \leq 2.4 \\
\frac{29.99-x}{29.99-24.99} \text { if } 2.4<x \leq 3.4 \\
1 \quad \text { if } x \geq 3.5 \\
\mu_{N o}=0 \text { if } x=0
\end{array}\right.
$$

Using the triangular membership, Equations $13-18$ show the membership functions used for the fuzzification of attribute SM.

$$
\begin{gathered}
\text { if } x=0 \\
S M(x)=\left\{\begin{array}{cc}
0 & \frac{x-1}{18.4-1} \text { if } 0 \leq x \leq 6 \\
\frac{24.99-x}{24.99-18.5} \text { if } 6<x \leq 12 \\
\frac{29.99-x}{29.99-24.99} \text { if } 12<x \leq 24 \\
1 \quad \text { if } x>24
\end{array}\right. \\
\mu_{\text {No }}=0 \text { if } x=\begin{array}{cc}
0 & \text { if } x<1
\end{array} \\
\mu_{\text {Low }}=\left\{\begin{array}{cc}
0 & \text { if } x>6 \\
\frac{x-1}{2.5} \text { if } & 1 \leq x \leq 3 \\
\frac{6-x}{2.5} \text { if } 3 \leq x \leq 6 & \text { if } x<6
\end{array}\right. \\
0.55 \quad \text { if } x>12
\end{gathered}
$$

$$
\mu_{\text {Low }}=\left\{\begin{array}{rrr}
0 & \text { if } x<0 \\
\frac{x}{0.7} \text { if } & 0<x<0.7 \\
\frac{1.4-x}{0.7} \text { if } & 0.7 \leq x \leq 1.4 \\
0.43 & \text { if } x>1.4
\end{array}\right.
$$

$$
\mu_{\text {Medium }}=\left\{\begin{array}{lr}
0 & \text { if } x<1.4 \\
\frac{x-1.4}{0.45} \text { if } & 1.4 \leq x \leq 1.85 \\
\frac{2.4-x}{0.45} \text { if } & 1.85<x \leq 2.4 \\
0.67 & \text { if } x>2.4
\end{array}\right.
$$

$$
\begin{gathered}
\mu_{\text {Normal }}=\left\{\begin{array}{lr}
0 & \text { if } x<2.4 \\
\frac{x-2.4}{0.5} \text { if } & 2.4 \leq x \leq 2.9 \\
\frac{3.4-x}{0.5} \text { if } & 2.9<x \leq 3.4 \\
0.72 & \text { if } x>3.4
\end{array}\right. \\
\mu_{\text {High }}=\left\{\begin{array}{lr}
0 & \text { if } x<3.5 \\
1 & \text { if } x \geq 3.5
\end{array}\right.
\end{gathered}
$$

\section{C) Fuzzy Inference}

The fuzzy inference system is a popular computing framework based on the concepts of fuzzy set theory, fuzzy If-Then rules, and fuzzy reasoning. The set of If-Then rules can be generated by domain experts or inferred from data. 
Olatunji K. A et al., International Journal of Information Systems and Computer Sciences, 7(5), September - October 2018, 25-33

An IF-THEN rule based is then computed for the premise and applied to obtain the stroke risk level. Consider the following definitions

$\mathrm{A}=\mathrm{IF}$ 'SM' is moderate AND 'BMI' is High AND ' $\mathrm{EX}$ ' is Low AND 'BP' is normal THEN 'SK' is low

$\mathrm{B}=\mathrm{IF}$ 'SM' is VeryHigh AND 'BMI' is High AND 'EX' is Low AND 'BP' is VeryHigh THEN 'SK' is High

where $\quad \mathrm{SM}=$ Smoking; $\quad \mathrm{BMI}=$ Body Mass Index; EX=Exercise; BP=Blood Pressure and SK=Stroke Risk.

Some of the rules were presented in table 5 .
The 'min' composition rules which is based on the principle of fuzzy logic operator 'and' is adopted. If there are rules ' $\mathrm{R}_{\mathrm{k}}$ ', $\mathrm{k}=$, $2 \ldots \mathrm{n}$, then the output signals of the rules ' $\mathrm{R}_{\mathrm{k}}$ ' is represented by the set;

$\left\{0\left(R_{1}\right)\right.$, then $\left.0\left(R_{k}\right), \ldots 0\left(R_{n}\right)\right\}$.

The aggregated output signal is computed by;

$$
\min \left\{0\left(\mathrm{R}_{1}\right) \text {, then } 0\left(\mathrm{R}_{\mathrm{k}}\right), \ldots 0\left(\mathrm{R}_{\mathrm{n}}\right)\right\} \text {. }
$$

It is noted that $0\left(\mathrm{R}_{\mathrm{k}}\right)<=1.0 ; \mathrm{k}=1,2 \ldots \mathrm{n}$,

Table 5: IF...THEN Rules for Prevention of Stroke

\begin{tabular}{|l|l|l|l|l|l|}
\hline \multirow{2}{*}{$\begin{array}{l}\text { RULE } \\
\text { NO }\end{array}$} & \multicolumn{4}{|c|}{ IF } & THEN \\
\cline { 2 - 6 } & SM & BMI & EX & BP & STROKE RISK \\
\hline 1 & Moderate & High & Low & Normal & Low \\
\hline 2 & VeryHigh & High & Low & VeryHigh & High \\
\hline 3 & No & High & Low & Normal & Low \\
\hline 4 & Low & Low & Moderate & Normal & Low \\
\hline 5 & Moderate & High & Low & Normal & Low \\
\hline 6 & Moderate & High & No & Normal & Low \\
\hline 7 & Low & Low & Low & Low & Low \\
\hline 8 & Low & Low & Medium & Low & Low \\
\hline 9 & Low & Low & Normal & Low & Low \\
\hline 10 & Low & Low & High & Low & Low \\
\hline
\end{tabular}

\section{D) Defuzzification}

The reasoning mechanism performs the inference procedure upon the rules and given facts to derive a reasonable conclusion. This is achieved by combining (through a specific T-norm operator, usually multiplication or $\mathrm{min}$ ) the membership values on the premise part to get firing strength (weight) of each rule. Qualified consequent (either fuzzy or crisp) of each rule is generated depending on the firing strength. These qualified consequents are then aggregated to produce a crisp output. The crisp output from the fuzzy logic system is displayed as the result. Also, management and prevention information from the Knowledge-base is then generated based on the set of input from the user and are also displayed as recommendation alongside the diagnosis result.

$$
\operatorname{Min}\left\{1, \mu_{A}(x)+\mu_{B}(x)\right\}
$$

where $\mu_{A}(x)$ and $\mu_{B}(x)$ is the set of all input parameters.

\section{E) Decision Support Engine}

Basically, decision support engine of this system enforces a standard which serves as a basis for the analysis of all the output reports of the inference engine. The inference engine in this system is based upon built knowledge base.

\section{CONCLUSION}

At the end of this research, a fuzzy-based expert system for prevention of stroke was designed. Patients data were collected and the risk factors BMI, SM, BP and EX serves as an input to the fuzzy logic to predict the occurrence of stroke. The designed system is expected to save a lot of lives i.e. by preventing stroke from happening or reoccurring again if implemented. It should be noted that the system was not developed to substitute human medical practitioners but rather help people with better understanding of Stroke.

\section{REFERENCES}

1. Amani Hessah. Medical Expert System" King Saud University College of Computer \& Information Sciences Department of Computer Science Second Semester 1427/1428 AH pp2, 2007. Retrieved 30/03/2016

2. Anindito, Bens Pardamean, Robby Christian and Bahtiar Saleh Abbas. Expert-System Based Medical Stroke Prevention. Journal of Computer Science 9 (9): ISSN: 1549-3636, pages 1099-1105, 2013.

3. J. Bamford, P. Sandercock, M. Dennis, J. Burn, C. Warlow. Classification and natural history of clinically identifiable subtypes of cerebral infarction. Lancet 1991, 337(8756):1521-1526, PubMedView Article. https://doi.org/10.1016/0140-6736(91)93206-O 
Olatunji K. A et al., International Journal of Information Systems and Computer Sciences, 7(5), September - October 2018, 25-33

4. L. R. Caplan, D. B. Hier, D'Cruz. Cerebral embolism in the Michael Reese Stroke Registry. Stroke 1983;14:530536 https://doi.org/10.1161/01.STR.14.4.530

5. V. L. Feigin. When Lightning Strikes: An Illustrated Guide to Stroke Prevention and Recovery. Kai Tiaki Nursing New Zealand, 10: 23-23, 2004.

6. M. A. Foulkes, P. A. Wolf, T. R. Price, J. P. Mohr, D. B. Hier. The Stroke Data Bank: Design, methods, and baseline characteristics. Stroke 1988;19:547-554 https://doi.org/10.1161/01.STR.19.5.547

7. Hakan Ay. A Computerized Algorithm for Etiologic Classification of Ischemic Stroke-The Causative Classification of Stroke System. America Stroke Association, 2007.

8. G. J. Hankey, and C. P. Warlow. Treatment and secondary prevention of stroke: Evidence, costs and effects on individuals and populations. Lancet, 354: 1457-1463. PMID: 10543686, 1999. https://doi.org/10.1016/S0140-6736(99)04407-4

9. Klaus Spitzer. The MICROSTROKE Expert System for Stroke Type Diagnosis. http://www:stroke1989; 20: 1353-1356.pdf, Retrieved 29/01/2016

10. Lee C. Ventola. Mobile Devices and Apps for Health Care Professionals: Uses and Benefits. US National library of medicine National Institute of Health, Available from https://www.nationalinstituteofhealth.com, 2014. Retrieved 30/12/2015,

11. J. P. Mohr, L. R. Caplan, J. W. Melski, R. J. Goldstein, G. W. Duncan, J. P. Kistler, M. S. Pessin, H. L. Bleich. The Harvard Cooperative Stroke Registry: A prospective registry. Neurology 1978;28:754-762 https://doi.org/10.1212/WNL.28.8.754

12. NHBPEP. The seventh report of the joint national committee on prevention, detection, evaluation and treatment of high blood pressure. National Institutes of Health, 2004. Retrieved 20/04/2016

13. Ruijin Ji. Web-based tool for dynamic functional outcome after acute ischemic stroke and comparison with existing models. BMC Neurology, 2014. Retrieved 26/01/2016

14. R. L.Sacco, R. Gan, B. Boden-Albala, I.F. Lin, D.E. Kargman and W.A. Hauser. Leisure-time physical activity and ischemic stroke risk: The Northern Manhattan stroke study. Stroke. 29: 380-387. PMID: 9472878, 1998. Retrieved 19/04/2016

15. Silke Walter. Mobile stroke.pdf. The lancet.com, 2012. Retrieved 30/01/2016 